\title{
Observation of trapping and release of carriers in InGaAs/GaAs quantum dots by ultrafast THz spectroscopy
}

Porte, Henrik; Jepsen, Peter Uhd; Daghestani, Nart; Rafailov, Edik; Turchinovich, Dmitry

Published in:

IEEE LEOS Annual Meeting Conference Proceedings, 2009. LEOS '09.

Link to article, DOI:

10.1109/LEOS.2009.5343415

Publication date:

2009

Document Version

Publisher's PDF, also known as Version of record

Link back to DTU Orbit

Citation (APA):

Porte, H., Jepsen, P. U., Daghestani, N., Rafailov, E., \& Turchinovich, D. (2009). Observation of trapping and release of carriers in InGaAs/GaAs quantum dots by ultrafast THz spectroscopy. In IEEE LEOS Annual Meeting Conference Proceedings, 2009. LEOS '09. (pp. ThD1). IEEE Press. https://doi.org/10.1109/LEOS.2009.5343415

\section{General rights}

Copyright and moral rights for the publications made accessible in the public portal are retained by the authors and/or other copyright owners and it is a condition of accessing publications that users recognise and abide by the legal requirements associated with these rights.

- Users may download and print one copy of any publication from the public portal for the purpose of private study or research.

- You may not further distribute the material or use it for any profit-making activity or commercial gain

- You may freely distribute the URL identifying the publication in the public portal 


\title{
Observation of Trapping and Release of Carriers in InGaAs/GaAs Quantum Dots by Ultrafast $\mathrm{THz}$ Spectroscopy
}

\author{
Henrik Porte*, Peter Uhd Jepsen*, Nart Daghestani ${ }^{\dagger}$, Edik Rafailov $^{\dagger}$, and Dmitry Turchinovich* \\ *DTU Fotonik - Department of Photonics Engineering, Technical University of Denmark, DK-2800 Kgs. Lyngby, Denmark \\ Email: dmtu@ fotonik.dtu.dk \\ ${ }^{\dagger}$ School of Engineering, Physics and Mathematics, University of Dundee, Dundee DD1 4HN, UK
}

\begin{abstract}
Depending on the photoexcitation wavelength, we either observe the trapping of the free carriers into quantum dots, or release of carriers from quantum dot ground state into conducting states of the quantum dot sample.
\end{abstract}

\section{INTRODUCTION}

Ultrafast carrier dynamics processes in semiconductor quantum dots (QDs), such as trapping and release of carriers, is important for operation of many optoelectronic devices such as QD lasers, QD infrared photodetectors (QDIPs), and QD semiconductor saturable absorber mirrors (QD SESAMs). In order to observe these processes, we employed an optical pump - THz probe technique [1]. Tunable optical pump pulses were used to excite the carriers either into the conducting barriers, or resonantly into the insulating QD ground state (GS). The $\mathrm{THz}$ probe pulses are sensitive to the presence of the free carriers, but are not attenuated by the carriers localized in the QDs. Thus, by using an optical pump $\mathrm{THz}$ probe technique we were able to monitor the population dynamics of the conducting states (CS) of the QD sample after photoexcitation (see Fig. 1(a)).
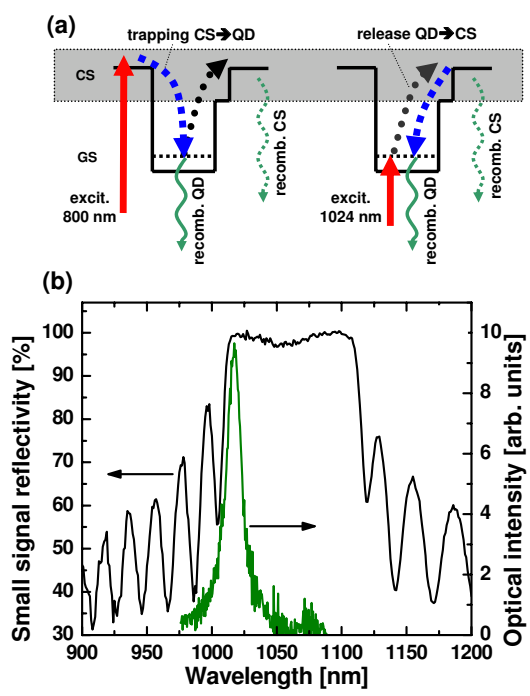

Fig. 1. a) Carrier dynamics processes in photoexcited QDs. CS - conducting state. GS - QD ground state. (b) Small signal reflectivity and room temperature optical emission spectra at $800 \mathrm{~nm}$ excitation of the studied QD SESAM.

\section{SAMPLE, EXPERIMENTAL RESULTS, AND DISCUSSION}

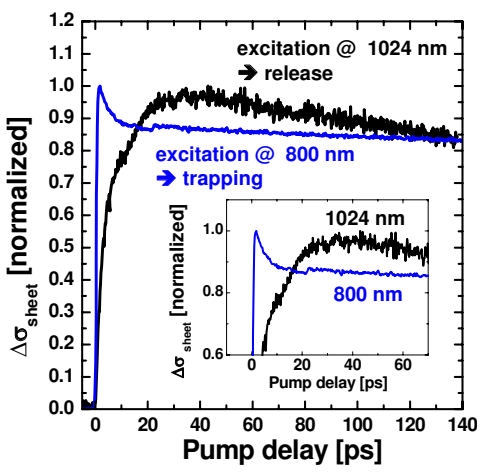

Fig. 2. Normalized $\Delta \sigma_{\text {sheet }}$ traces for QD GS and barrier excitation with the pump wavelength and fluence of $1024 \mathrm{~nm}$ and $22.8 \mu \mathrm{J} / \mathrm{cm}^{2}$, and $800 \mathrm{~nm}$ and $1.18 \mu \mathrm{J} / \mathrm{cm}^{2}$, respectively. Inset: zoomed-in traces.

As a sample we used a QD SESAM, grown by molecular beam epitaxy (MBE). It had 15 layers of $\operatorname{In}_{0.5} \mathrm{Ga}_{0.5} \mathrm{As} / \mathrm{GaAs}$ QDs separated by GaAs spacers, grown on top of the $\mathrm{Al}_{0.9} \mathrm{Ga}_{0.1} \mathrm{As} / \mathrm{GaAs}$ Bragg reflector. The total thickness of QD and spacer region was $611 \mathrm{~nm}$. The sample was grown on a $0.5-\mathrm{mm}$ thick semi-insulating GaAs substrate. The presence of a Bragg reflector in the sample was important for our experiment, since it was blocking the propagation of the pump light at resonant QD GS excitation into the GaAs substrate, thus preventing the generation of free carriers there by twophoton absorption mechanism. The small-signal reflectivity spectrum of the studied QD SESAM, as well as the roomtemperature optical emission spectrum at 800 -nm excitation, indicating the energetic position of the QD GS, are shown in Fig. 1(b).

An output of a regenerative Ti:Sapphire femtosecond amplifier, operating at a repetition rate of $1 \mathrm{kHz}$, and generating $45 \mathrm{fs}$ pulses at $800 \mathrm{~nm}$ central wavelength, was split into three beans. One beam was used as a pump pulse: either directly, for excitation of the barrier states of the QDs at $800 \mathrm{~nm}$; or after frequency conversion in an optical parametric amplifier (OPA) to $1024 \mathrm{~nm}$, for resonant excitation of the QD GS. Two other beams were used to drive the standard nonlinear crystal - based $\mathrm{THz}$ time-domain spectrometer, with a sample 
positioned at its focal point [1]. The $\mathrm{THz}$ probe pulses were approximately 300-fs long, and had a useful spectrum of 0.5-2.5 THz. All our experiments were performed at room temperature.

As discussed above, the THz probe pulses are only sensitive to the population of the CS of the sample, but not to the population of the QDs. Both optical pump and $\mathrm{THz}$ probe pulses were normally incident onto a QD sample. Thus, the in-plane conductivity of the sample was probed with $\mathrm{THz}$ pulses. The relative transmission of the probe $\mathrm{THz}$ pulse can be quantified and converted into a photoinduced sheet conductivity $\Delta \sigma_{\text {sheet }}$ [2]. The typical results of the photoconductivity dynamics of the sample depending on the excitation wavelength are shown in Fig. 2. In case of barrier excitation at $800 \mathrm{~nm}$, an instantaneous rise of photoconductivity due to direct injection of the free carriers, is followed by a very fast decay, associated to the trapping of these carriers into the QDs, and a much slower component caused by the conductivity in Type-II Bragg reflector, accessible to the pump light at this wavelength. If the QDs were resonantly excited in to the GS at 1024 $\mathrm{nm}$, the maximum in the photoconductivity is reached only approximately $35 \mathrm{ps}$ after the photoexcitation. We attribute this onset of the population of the CS to the release of the carriers from the QD GS to higher-energy CS, most likely driven by a large difference of densities of states between the QD GS and CS.

The maxima of $\Delta \sigma_{\text {sheet }}$ dynamics for $800 \mathrm{~nm}(1024 \mathrm{~nm})$ excitation show linear (sub-linear) dependency on the pump fluence, as shown in Fig. 3, precisely as expected from a onephoton photoexcitation process. The saturation of maximum $\Delta \sigma_{\text {sheet }}$ for $1024 \mathrm{~nm}$ pump revealed a saturation fluence value of $F_{s}=40 \mu \mathrm{J} / \mathrm{cm}^{2}$ [3], in reasonable agreement with the alloptical measurement on similar SESAMs [4].

The $\Delta \sigma_{\text {sheet }}$ dynamics for $1024 \mathrm{~nm}$ resonant QD GS excitation does not change significantly with increase in pump fluence. However, in the case of barrier excitation at 800 $\mathrm{nm}$, we observed a slow-down of a fast decay component of $\Delta \sigma_{\text {sheet }}$ dynamics, associated with trapping of the carriers into QDs. We attribute this slowing down to filling up of the QD (trap) states with growth in excitation fluence [5], [6], [7]. In order to quantitatively estimate the trapping time, we have measured the $10 \%$ decay time constant, which shows nearlinear growth with $800 \mathrm{~nm}$ excitation fluence (see Fig. 4).

\section{CONCLUSION}

Using optical pump - THz probe technique, we were able to demonstrate the difference in ultrafast conductivity dynamics of a QD sample, depending on what state is optically excited: the QD GS or the barrier states. The observed dynamics can be explained by the dominating carrier release or carrier trapping processes in the QDs, respectively.

\section{ACKNOWLEDGMENT}

We would like to thank Danish Advanced Technology Foundation (HTF) and EU FP7 Programme (FAST-DOT) grant 224338 for financial support.
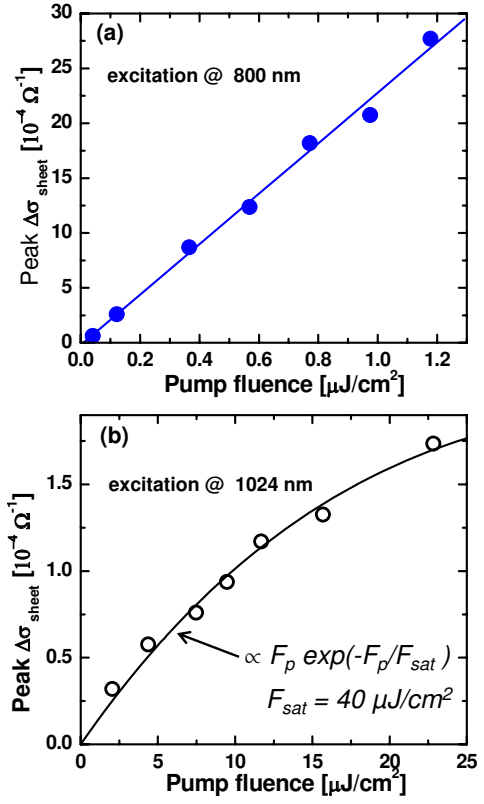

Fig. 3. Maximum of $\Delta \sigma_{\text {sheet }}$ traces as a function of pump fluence for (a) barrier excitation at $800 \mathrm{~nm}$. Solid line: linear fit; and (b) resonant QD GS excitation at $1024 \mathrm{~nm}$. Solid line: fit to the saturable absorption function with saturation fluence $F_{s}=40 \mu \mathrm{J} / \mathrm{cm}^{2}$.

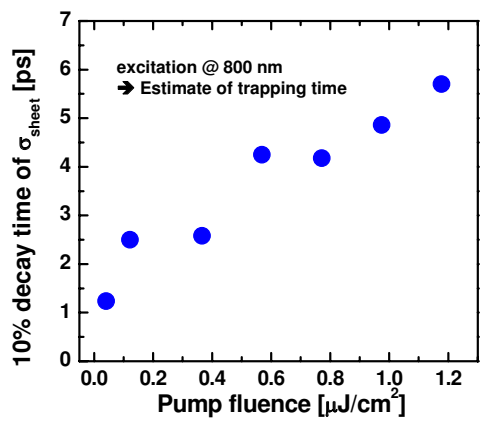

Fig. 4. Estimate of the QD trapping time: $10 \%$ decay time constant of the QD SESAM $\Delta \sigma_{\text {sheet }}$ traces, as a function of $800-\mathrm{nm}$ pump fluence.

\section{REFERENCES}

[1] M. Schall and P. Uhd Jepsen, "Photoexcited GaAs surfaces studied by transient terahertz time-domain spectroscopy," Opt. Lett. 25, 13 (2000).

[2] K. P. H. Lui and F. A. Hegmann, "Ultrafast carrier relaxation in radiationdamaged silicon on sapphire studied by optical-pumpterahertz-probe experiments," Appl. Phys. Lett. 78, 3478 (2001).

[3] M. Haiml et al., "Optical characterization of semi- conductor saturable absorbers," Appl. Phys. B: Lasers Opt. 79, 331 (2004).

[4] A. A. Lagatsky et al.,"Low-loss quantum-dot-based saturable absorber for efficient femtosecond pulse generation," Appl. Phys. Lett. 91, 231111 (2007).

[5] D. Turchinovich et al.,"InAs/GaAs quantum dots as efficient free carrier deep traps ," phys. stat. sol. (c) 0, 1556 (2003).

[6] D. G. Cooke et al.,"Anisotropic photoconductivity of InGaAs quantum dot chains measured by terahertz pulse spectroscopy," Appl. Phys. Lett. 85, 3839 (2004).

[7] P. Uhd Jepsen et al.,"Ultrafast carrier trapping in microcrystalline silicon observed in optical pumpterahertz probe measurements," Appl. Phys. Lett. 79, 1291 (2001). 